Marquette University

e-Publications@Marquette

$10-2017$

\title{
Predictors of Comorbid Eating Disorders and Association with Other Obsessive-Compulsive Spectrum Disorders in Trichotillomania
}

\author{
Erica Greenberg \\ Massachusetts General Hospital/Harvard Medical School \\ Jon E. Grant \\ University of Chicago \\ Erin E. Curley \\ Massachusetts General Hospital/Harvard Medical School \\ Christine Lochner \\ Stellenbosch University \\ Douglas W. Woods \\ Marquette University, douglas.woods@marquette.edu
}

See next page for additional authors

Follow this and additional works at: https://epublications.marquette.edu/psych_fac

Part of the Psychology Commons

\section{Recommended Citation}

Greenberg, Erica; Grant, Jon E.; Curley, Erin E.; Lochner, Christine; Woods, Douglas W.; Tung, Esther S.; Stein, Dan J.; Redden, Sarah A.; Scharf, Jeremiah M.; and Keuthen, Nancy, "Predictors of Comorbid Eating Disorders and Association with Other Obsessive-Compulsive Spectrum Disorders in Trichotillomania" (2017). Psychology Faculty Research and Publications. 289.

https://epublications.marquette.edu/psych_fac/289 


\section{Authors}

Erica Greenberg, Jon E. Grant, Erin E. Curley, Christine Lochner, Douglas W. Woods, Esther S. Tung, Dan J. Stein, Sarah A. Redden, Jeremiah M. Scharf, and Nancy Keuthen 
Marquette University

e-Publications@Marquette

\section{Psychology Faculty Research and Publications/College of Arts and Sciences}

This paper is NOT THE PUBLISHED VERSION; but the author's final, peer-reviewed manuscript. The published version may be accessed by following the link in the citation below.

Comprehensive Psychiatry, Vol. 78 (October, 2017): 1-8. DOI. This article is (C) Elsevier (WB Saunders) and permission has been granted for this version to appear in e-Publications@Marquette. Elsevier (WB Saunders) does not grant permission for this article to be further copied/distributed or hosted elsewhere without the express permission from Elsevier (WB Saunders).

\section{Predictors of Comorbid Eating Disorders and Association with Other Obsessive-Compulsive Spectrum Disorders in Trichotillomania}

\section{Erica Greenberg}

Department of Psychiatry, Massachusetts General Hospital/Harvard Medical School, Boston, MA Jon E. Grant

Department of Psychiatry and Behavioral Neuroscience, University of Chicago, Chicago, IL

Erin E. Curley

Department of Psychiatry, Massachusetts General Hospital/Harvard Medical School, Boston, MA Christine Lochner

SU/UCT MRC Unit on Anxiety and Stress Disorders, Department of Psychiatry, Stellenbosch University, Cape Town, South Africa

Douglas W. Woods

Department of Psychology, Marquette University, Milwaukee, WI 
Esther S. Tung

Department of Psychology, Boston University, Boston, MA

Dan J. Stein

Department of Psychiatry and MRC Unit on Anxiety \& Stress Disorders, University of Cape Town, Cape Town, South Africa

Sarah A. Redden

Department of Psychiatry and Behavioral Neuroscience, University of Chicago, Chicago, IL Jeremiah M. Scharf

Department of Psychiatry, Massachusetts General Hospital/Harvard Medical School, Boston, MA Psychiatric \& Neurodevelopmental Genetics Unit, Center for Human Genetics Research, Massachusetts General Hospital/Harvard Medical School, Boston, MA

Nancy J. Keuthen

Department of Psychiatry, Massachusetts General Hospital/Harvard Medical School, Boston, MA

\section{Abstract}

Trichotillomania (TTM) and eating disorders (ED) share many phenomenological similarities, including ritualized compulsive behaviors. Given this, and that comorbid EDs may represent additional functional burden to hair pullers, we sought to identify factors that predict diagnosis of an ED in a TTM population. Subjects included 555 adult females (age range 18-65) with DSM-IV-TR TTM or chronic hair pullers recruited from multiple sites. $7.2 \%(\mathrm{~N}=40)$ of our TTM subjects met criteria for an ED in their lifetime. In univariable regression analysis, obsessive-compulsive disorder (OCD), Yale-Brown Obsessive Compulsive Scale (Y-BOCS) worst-ever compulsion and total scores, certain obsessive-compulsive spectrum disorders, anxiety disorder, attention-deficit/hyperactivity disorder (ADHD), and substance disorder all met the pre-specified criteria for inclusion in the multivariable analysis. In the final multivariable model, diagnosis of OCD (OR: $5.68,95 \% \mathrm{Cl}: 2.2-15.0$ ) and diagnosis of an additional body-focused repetitive behavior disorder (BFRB) (OR: 2.69, 95\% Cl: 1.1-6.8) were both associated with increased risk of ED in TTM. Overall, our results provide further support of the relatedness between ED and TTM. This finding highlights the importance of assessing for comorbid OCD and additional BFRBs in those with TTM. Future research is needed to identify additional predictors of comorbid disorders and to better understand the complex relationships between BFRBs, OCD and EDs.

\section{Introduction}

Trichotillomania (TTM) is a disorder of repetitive hair pulling, characterized by hair loss, inability to stop pulling and functional impairment or distress ${ }^{[1]}$. Prevalence estimates vary from $0.6 \%$ of the population ${ }^{[2]}$ to $3.4 \%$ of women and $1.5 \%$ of men ${ }^{[3]}$, depending on how the diagnosis was made. While the gender distribution in the community varies, the clinical TTM population tends to be dominated by women $[4]$.

TTM is categorized as an obsessive-compulsive and related disorder in the DSM-5 ${ }^{[1]}$. It is also considered to be an obsessive-compulsive spectrum disorder (OCSD) along with OCD, body dysmorphic disorder (BDD), hoarding disorder, tic disorders, and excoriation (skin-picking) disorder (SPD). TTM and other OCSDs share phenomenology, neurobiology (i.e. fronto-cortico-striatal circuitry dysfunction), 
common comorbidities and familial/genetic features [5], [6], [7], [8], [9]. These disorders also share genetic vulnerability and occur in one another with increased prevalences $[10]$.

Comorbid eating disorders (EDs) are not uncommon in TTM. In 1991, Christenson et al. found that about $20 \%$ of chronic hair-pullers had eating disorders (comprised of bulimia and "eating disorder not otherwise specified") [11]. Houghton and et al. also reviewed the prevalence of EDs in smaller TTM populations, and found that anorexia nervosa ranged from $1.6 \%-5 \%$, bulimia nervosa ranged from $2 \%-$ $14 \%$, and binge eating disorder ranged from $6 \%-10.2 \%$ [9] (reflecting elevated prevalence rates of EDs than in the general population).

Other disorders are also frequently comorbid in TTM. Houghton et al. found that $78.8 \%$ of TTM subjects met criteria for at least one lifetime comorbid psychiatric condition, including OCD (5\%-30\%), major depressive disorder (32\%-55\%), generalized anxiety disorder (16.6\%-32\%), alcohol use disorder (2.6\%-19.4\%), and substance abuse disorder (8.3\%-22\%) [9]. SPD prevalence rates range from $10 \%-$ $34 \%{ }^{[12]}$. In general, anxiety disorders are common in TTM and are thought by some researchers to be central in both the etiology and maintenance of eating disorders [13]. The prevalence of anxiety disorders, including simple phobia (now known as specific phobia), avoidant disorder, separation anxiety, post-traumatic stress disorder (PTSD), and OCD is $37 \%$ [14].

Many researchers believe that like TTM, EDs should be considered as part of the obsessive-compulsive spectrum [15], [16], [17], [18]. OCSDs and EDs are both characterized by difficulties inhibiting repetitive behaviors and a subjective sense of compulsion [6]. TTM and EDs are also part of a small group of disorders thought to have both compulsive and impulsive components ${ }^{[5]}$. In addition to sharing similar phenomenology and functionality ${ }^{[18]}$, they may also share pathophysiological mechanisms, including cortico-striatal dysfunction $[15]$. There are elevated prevalences of OCD within ED populations (11\%$41 \%)[19]$, and of EDs within OCD populations (11\%-42\%) [15], [17]. A recent study by Cederlof et al. showed that females and males with OCD had respective 16-fold and 37-fold increased risks of having a comorbid diagnosis of anorexia nervosa $[17]$. Given that OCD is elevated in relatives of probands with EDs ${ }^{[16]}$ and the risk for EDs is elevated in relatives of probands with OCD ${ }^{[17]}$, Cederlof et al. concluded that the comorbid pattern is at least in part due to shared genetic factors ${ }^{[17]}$. This observation is important because the presence of obsessive-compulsive symptomatology has been positively associated with the severity of the ED ${ }^{[19]}$. It also points to the likelihood that OCD may be a predictive factor of the presence of an ED within TTM populations.

Despite many similarities/correlations, there has been limited research thus far on the specific relationships between EDs and TTM $[5]$, and what factors may be predictive of having an ED within a TTM population. Recently, Keuthen et al. looked at the predictive factors of having an OCD diagnosis within a TTM population, and found that having an ED diagnosis was associated with greater risk for OCD [20]. In 2011, Zucker et al. assessed the predictive factors of hair pulling within an ED population [5]. Five percent of their female ED population had evidence of repetitive hair pulling ${ }^{[5]}$. The significant predictors of hair pulling were (OCD-related) compulsive features and trait anxiety ${ }^{[5]}$.

Given reported comorbidities and traits commonly associated with TTM and EDs, we were tasked with choosing variables a priori that may lead to increased risk of having an ED within a TTM population. In 
addition to including OCD diagnosis as a variable, measures of compulsion (i.e. $\underline{\text { Y-BOCS }}$ sub-score) were included. Given the preponderance of the association of anxiety disorders and depression with EDs, generalized anxiety, specific phobia, 'any anxiety disorder,' the Beck Anxiety Inventory (BAI) score, and major depressive disorder were included [14], [21]. ED probands tend to have more tic disorders and substance abuse compared to controls ${ }^{[16]}$, and so tic disorders and substance abuse were included as well. Other body-focused repetitive behavior disorders (BFRBs), such as skin-picking and severe nail biting, were included as potential factors given their strong relationship to TTM and OCD. Lastly, given the impulsive components found in TTM and ED, disorders known for increased impulsivity, such as $\underline{A D H D}$ and bipolar disorder (in addition to substance abuse disorders) were included $\underline{[22]}$.

Ultimately, determining which factors are more predictive of having an ED within a TTM population is important to the clinician who works with patients with TTM. The combination of TTM and EDs represents an increased functional burden for the patient, in addition to EDs themselves having a high morbidity/mortality rate [23], [24]. Additionally, individuals with EDs may be reluctant to disclose their condition, and so having a more accurate "pre-test probability" could help identify sufferers [25].

\section{Methods}

\subsection{Participants}

Five-hundred fifty-five adult female hair pullers were recruited from both treatment and nontreatment studies at Massachusetts General Hospital $(n=232)$, the University of Chicago $(n=40)$, the University of Minnesota ( $n=109)$, the University of Wisconsin-Milwaukee $(n=97)$, and Stellenbosch University $(n=77)$ between 2006 and 2015. All subjects were between 18 and 65 years of age and met diagnostic criteria for DSM-IV-TR ${ }^{[2]}$ TTM or chronic hair pulling (CHP). The latter was defined by meeting $D S M-I V-T R$ TTM criteria except for tension before pulling or when attempting to resist (formally criterion B) and/or pleasure, relief, or gratification when pulling (formally criterion C) and is considered analogous to DSM-5 TTM criteria.

Participant data was drawn from TTM genetics, imaging, and treatment studies and as a result, some differences in site exclusion criteria were present. Subjects from Massachusetts General Hospital and Stellenbosch University were excluded if they met criteria for a lifetime diagnosis of psychosis, autism, or mental retardation. Subjects at the University of Chicago and the University of Minnesota were excluded if they were pregnant or met lifetime criteria for bipolar disorder or psychosis. Subjects at the University of Wisconsin-Milwaukee, University of Chicago and University of Minnesota were excluded if they had unstable medical illness, bipolar disorder, psychotic disorder, dementia, current substance dependence, mental retardation or pervasive developmental disorder, or were evaluated to be a suicide risk. Participants were also excluded if they were pregnant or lactating, had an estimated $\mathrm{IQ}<85$, had a head injury/neurological disorder (i.e. epilepsy), had a medication change within the prior 8 weeks or were currently in psychotherapy for another condition. Of note, other analyses have been conducted on this dataset in part and/or in its entirety [20].

Participants were recruited from multiple sources including the TLC Foundation for BFRBs (the national advocacy organization for body-focused repetitive behaviors), local mental health clinics, hospital 
intranets, and flyers in the community. Study approval was received from the Institutional Review Boards of all relevant institutions prior to study initiation. All subjects gave informed consent prior to study participation. Modest financial compensation was offered to participants at each study site with some variability across the sites. Data were de-identified according to the Safe Harbor method for deidentification prior to data sharing $(\S 164.514(b))$ [26].

\subsection{Assessment materials}

Semi-structured interviews were utilized for the diagnosis of TTM or CHP and assessment of psychiatric comorbidities and family history. The diagnostic interviews were performed by MD or PhD faculty at each site or by study staff trained to reliability on the structured interviews. A self-report measure was used to assess TTM and OCD severity. The relevant scales are described below.

\subsubsection{Semi-structured interviews}

\subsubsection{The structured clinical inventory for DSM-IV axis I disorders, patient edition ${ }^{[27]}$ (SCID-I/P)}

The SCID-I/P is a structured clinical interview with well-accepted reliability and validity. It is currently considered the gold standard for psychiatric diagnosis. The SCID was used at all sites to assess for current and lifetime eating, other comorbid and exclusionary disorders. The EDs diagnosed in this population include anorexia nervosa, bulimia nervosa and binge eating disorder.

\subsubsection{Schedule for affective disorders and schizophrenia-present and lifetime version [28] (K-SADS-PL)}

The K-SADS-PL is a semi-structured diagnostic interview designed to assess current and past episodes of psychopathology in children and adolescents utilizing both DSM-III-R and DSM-IV diagnostic criteria. The attention deficit hyperactivity disorder (ADHD) module was used at Massachusetts General Hospital, University of Chicago, University of Minnesota, and Stellenbosch University for assessment of ADHD (inattention, hyperactivity, and impulsivity) in adults.

\subsubsection{The trichotillomania diagnostic inventory-revised $[29]$ (TDI-R)}

The TDI-R is a clinician-based semi-structured interview modeled after the SCID and is an updated version of the original TDI to be consistent with DSM-IV-TR TTM criteria. The TDI-R consists of 3-point ratings of responses to six items. It was utilized in the present study to diagnose TTM or CHP.

\subsubsection{The skin picking diagnostic inventory $[30]$ (SPDI)}

The SPDI is a clinician-administered interview for skin picking that is analogous to the TDI-R. The diagnostic criteria for SPD and CSP (a diagnosis largely parallel to DSM-5 SPD) are modeled after DSMIV criteria for TTM and CHP. The SPDI consists of 3-point ratings of responses to six items assessing these diagnostic criteria. The SPDI was used to assess for the presence of SPD and CSP. 


\subsubsection{The Schedule for Tourette's Syndrome and other behavioral syndromes [31,32] (STOBS)}

This interview includes symptom checklists and severity ratings based on the Yale Brown Obsessive Compulsive Scale (Y-BOCS) for documentation of OC symptoms and the Yale Global Tic Severity Scale (YGTSS) for documentation of tics. It also has a BFRB section for the assessment of hair pulling, skin picking, and nail biting. OCD and Tourette's Syndrome (TS) were diagnosed using the DSM-IV-TR criteria. Those who endorsed repetitive nail biting that caused significant distress were deemed to have clinical levels of nail biting. The interviews have been shown to be both valid and reliable for the diagnosis of OCD, TS, and chronic tics [33]. The STOBS was used at Massachusetts General Hospital and Stellenbosch University to assess comorbid diagnoses of OCD, TS, chronic tics, and nail biting plus OCD severity.

\subsubsection{Self-reports scales}

\subsubsection{Beck anxiety inventory ${ }^{[34]}$ (BAI)}

This 21-item self-report measure asks participants to assess their anxiety symptoms during the past week on a four-point Likert scale from 0 to 3 . Higher scores indicate more severe anxiety. The BAI has demonstrated good internal consistency, with alpha coefficients of 0.90-0.92 [34]. This measure was used at Massachusetts General Hospital and University of Wisconsin-Milwaukee to assess anxiety severity.

\subsection{Statistical analyses}

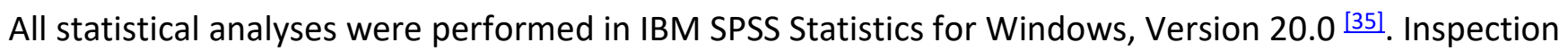
of variable plots suggested largely normal distributions for the majority of variables. Initial analyses were used to assess significance of candidate predictor variables. Univariable logistic regression was used to determine the odds ratios and $95 \%$ confidence intervals for categorical and continuous candidate predictors. Beta significance levels were set at 0.20 . Clinical predictors from the univariable analyses with $p<0.10$ were subsequently included in the final multivariable models predicting comorbid EDs in TTM/CHP. Age at interview was also included as a covariate in the final multivariable models to control for some participants not yet having passed through the risk periods for development of some comorbid disorders. In the final model, multivariable logistic regression was used and variables were considered to be independently associated with comorbid EDs at a threshold of $p<0.05$.

\subsection{Sample description}

The mean (SD) age of the participants was 32.6 (11.7) years. (see Table 1 for a description of the sample). Of the 40 individuals diagnosed with lifetime EDs, 10 were diagnosed with anorexia nervosa, 12 were diagnosed with bulimia nervosa, 14 were diagnosed with binge eating disorder, and 4 had non-specific diagnoses. 
Table 1. Sample characteristics.

Factor

n

Race

White/Caucasian

Black/African American

Asian

Multi-Racial

Other

Ethnicity

Hispanic/Latino

10

1.8

Marital status
474

85.4

23

4.1

8

1.4

18

3.2

1.6
310

184

7

27

15

304

85

Comorbid lifetime diagnoses

Education

$\begin{array}{cc}\text { Separated } & 7 \\ \text { Divorced } & 27 \\ \text { Widowed } & 15\end{array}$

$142 \quad 25.6$

\footnotetext{
Graduate degree

High school or less

College or some college
}

$$
\text { OCD }
$$

SPD/chronic skin picking

Nail biting

Body-dysmorphic disorder

Eating disorder

Anxiety disorder

Substance use disorder

Tic disorder

Major depressive disorder

Bipolar disorder

1.3

4.9

2.7

$105 \quad 18.9$

$108 \quad 19.5$

$71 \quad 12.8$

$15 \quad 2.7$

$40 \quad 7.2$

$200 \quad 36.0$

$88 \quad 15.9$

$16 \quad 2.9$

$277 \quad 49.9$

$5 \quad 0.9$

$\mathrm{OCD}=$ obsessive-compulsive disorder $; \mathrm{SPD}=$ skin picking disorder; eating disorder $=$ anorexia nervosa, $\underline{\text { bulimia nervosa, }}$ and/or binge eating disorder; anxiety disorder = panic disorder without agoraphobia, panic disorder with agoraphobia, agoraphobia, specific phobia, social anxiety disorder, separation anxiety, general anxiety disorder, and post-traumatic stress disorder.

Mean (SD) onset of hair pulling was 12.7 (5.9) years. The mean (SD) score for Y-BOCS worst ever compulsions was 4.6/20 (5.6) and the mean (SD) score for Y-BOCS worst ever obsessions and compulsions was 8.7/40 (10.5). For those who met criteria for OCD, the mean (SD) score for Y-BOCS 
worst ever compulsions was 10.5/20 (3.8), and the mean (SD) score for Y-BOCS worst ever total was 20.2/40 (7.0). The mean (SD) total score for the BAI was 10.6/63 (9.5).

\section{Results}

\subsection{Univariable analyses}

In the univariable analyses predicting comorbid EDs in TTM/CHP, we investigated a number of variables including age, Y-BOCS worst ever compulsions score, Y-BOCS worst ever total score, BAI total score, diagnosis of $O C D$, diagnosis of GAD, diagnosis of any anxiety disorder, diagnosis of SPD/CSP, diagnosis of nail biting, diagnosis of an additional BFRB, diagnosis of BDD, diagnosis of $\underline{A D H D}$, diagnosis of a substance disorder, diagnosis of any tic disorder, diagnosis of MDD, and diagnosis of bipolar disorder (Table 1). "Any BFRB" included those with SPD/CSP and/or severe nail biting. Y-BOCS worst ever compulsions score, Y-BOCS worst ever total score, diagnosis of OCD, diagnosis of any anxiety disorder, diagnosis of an additional BFRB, diagnosis of BDD, diagnosis of ADHD, and diagnosis of a substance disorder each met the pre-specified criteria for inclusion in the multivariable analysis (Table 2).

Table 2. Univariable predictors of comorbid eating disorders in a TTM population.

\begin{tabular}{|c|c|c|c|c|}
\hline Continuous predictors & \multicolumn{2}{|c|}{ Mean (SD) } & OR $(95 \% \mathrm{Cl})$ & $p$-Value \\
\hline Age & \multicolumn{2}{|c|}{$33.25(11.68)$} & $1.00(0.98-1.03)$ & 0.74 \\
\hline Y-BOCS worst ever compulsion & \multicolumn{2}{|c|}{$8.65(6.09)$} & $1.15(1.07-1.24)$ & $<0.001$ \\
\hline Y-BOCS worst ever obsessions and compulsions & \multicolumn{2}{|c|}{$15.42(11.77)$} & $1.06(1.02-1.10)$ & 0.001 \\
\hline BAl total score & \multicolumn{2}{|c|}{$12.81(9.61)$} & $1.02(0.99-1.06)$ & 0.17 \\
\hline Categorical predictors & $\mathbf{N}(\%)$ & \multicolumn{2}{|r|}{ OR $(95 \% \mathrm{Cl})$} & $p$-Value \\
\hline OCD & $19(47.5)$ & \multicolumn{2}{|c|}{$4.47(2.30-8.67)$} & $<0.001$ \\
\hline Generalized anxiety disorder & $8(20.0 \%)$ & \multicolumn{2}{|c|}{$1.12(0.50-2.51)$} & 0.78 \\
\hline Any anxiety disorder & $21(52.5 \%)$ & \multicolumn{2}{|c|}{$2.06(1.08-3.92)$} & 0.03 \\
\hline SPD/CSP & $11(27.5 \%)$ & \multicolumn{2}{|c|}{$1.59(0.75-3.37)$} & 0.22 \\
\hline Nail biting & $5(12.5 \%)$ & \multicolumn{2}{|c|}{$0.94(0.35-2.56)$} & 0.91 \\
\hline SPD/CSP and/or severe nail biting & $16(40.0 \%)$ & \multicolumn{2}{|c|}{$1.99(0.91-4.32)$} & 0.08 \\
\hline Body dysmorphic disorder & $3(7.5 \%)$ & \multicolumn{2}{|c|}{$3.28(0.88-12.25)$} & 0.08 \\
\hline ADHD & $6(15.0 \%)$ & \multicolumn{2}{|c|}{$2.68(1.03-6.97)$} & 0.04 \\
\hline Substance use disorder & $12(30.0 \%)$ & \multicolumn{2}{|c|}{$2.62(1.26-5.48)$} & 0.01 \\
\hline Any tic disorder & $3(7.5 \%)$ & \multicolumn{2}{|c|}{$2.05(0.55-7.63)$} & 0.28 \\
\hline Major depressive disorder & $25(62.5 \%)$ & \multicolumn{2}{|c|}{$1.71(0.88-3.32)$} & 0.11 \\
\hline Bipolar disorder & $1(2.5 \%)$ & \multicolumn{2}{|c|}{$3.24(0.35-29.73)$} & 0.30 \\
\hline
\end{tabular}


$\mathrm{SD}=$ standard deviation; $\mathrm{OR}=\underline{\text { odds ratio }} ; \underline{\text { Y-BOCS }}=\underline{\text { Yale-Brown Obsessive Compulsive Scale; }} \mathrm{BAI}=\underline{\text { Beck Anxiety Inventory; }}$

$\mathrm{OCD}=$ obsessive-compulsive disorder; $\mathrm{SPD} / \mathrm{CSP}=$ skin picking disorder/chronic skin picking; $\underline{\mathrm{ADHD}}=\underline{\text { attention- }}$

deficit/hyperactivity disorder.

\subsection{Multivariable analyses}

Due to multicollinearity with diagnosis of OCD, Y-BOCS worst ever compulsions score (tolerance $=0.108 ; \mathrm{VIF}=0.282$ ) and $\mathrm{Y}$-BOCS worst ever total score (tolerance $=0.108 ; \mathrm{VIF}=0.282$ ) were not included in the final multivariable model. In the final multivariable model of comorbid EDs in TTM/CHP (Table 3), diagnosis of OCD (OR = 5.7, $\mathrm{Cl}$ [2.16-14.99], $p<0.001)$ and diagnosis of an additional $\mathrm{BFRB}(\mathrm{OR}=2.7, \mathrm{Cl}$ [1.07-6.76], $p=0.035)$ remained significant predictors of increased risk of comorbid EDs in TTM/CHP (Table 3).

Table 3. Multivariable predictors of comorbid eating disorders in a trichotillomania population.

$\begin{array}{clllllll}\text { Variable } & \text { B } & \text { S.E. } & \text { Wald } & \text { df } & p \text {-Value } & \text { OR (95\% CI) } \\ \text { Age } & 0.006 & 0.02 & 0.074 & 1 & 0.78 & 1.01(0.97-1.05) \\ \text { OCD } & 0.495 & 0.495 & 12.339 & 1 & <0.001 & 5.68(2.16-14.99) \\ \text { Any anxiety disorder } & 0.906 & 0.474 & 0.906 & 1 & 0.34 & 1.57(0.62-3.97) \\ \text { SPD/CSP and/or severe nail biting } & 0.989 & 0.47 & 4.423 & 1 & 0.04 & 2.69(1.07-6.76) \\ \text { Body dysmorphic disorder } & 1.43 & 0.825 & 3.002 & 1 & 0.08 & 4.18(0.83-21.06) \\ \text { ADHD } & 0.872 & 0.612 & 2.031 & 1 & 0.15 & 2.39(0.72-7.94) \\ \text { Substance use disorder } & 0.183 & 0.539 & 0.116 & 1 & 0.73 & 1.02(0.42-3.45) \\ \text { Constant } & -4.338 & 0.807 & 28.878 & 1 & <.001 & \end{array}$

$\mathrm{B}=$ beta coefficient; $\mathrm{S} . \mathrm{E} .=$ standard error; $\mathrm{df}=$ degrees of freedom; $\mathrm{OR}=$ odds ratio; $\mathrm{Cl}=$ confidence interval; $\mathrm{OCD}=$ obsessive-compulsive disorder; SPD/CSP = skin picking disorder/chronic skin picking; $\underline{A D H D}=\underline{\text { attention-deficit/hyperactivity }}$ disorder.

\section{Discussion}

This study examined the largest sample of clinically diagnosed individuals with TTM/chronic hair pulling to date. The results overall provide evidence to support the relatedness between TTM, OCD and EDs.

The prevalence of comorbidities in this cohort of individuals with trichotillomania was similar to that found in other studies examining prevalence ${ }^{[9]}$. Individuals with lifetime EDs made up $7.2 \%$ of this TTM population. While that proportion of TTM individuals with comorbid EDs is lower than the $20 \%$ found by Christenson et al. [11], it is consistent with the ED prevalence rates found in Houghton et al.'s more recent study in which the prevalence of anorexia nervosa, bulimia and binge eating disorders was $4.7 \%, 1.2 \%$ and $1.2 \%$, respectively ${ }^{[9]}$. The prevalence of OCD in this sample, $18.9 \%$, is in the $5 \%-30 \%$ range compiled by Houghton et al. [9]. The percentage of individuals with chronic skin-picking/SPD is consistent with prior findings as well.

From the univariable analysis, greater $\underline{\mathrm{Y} \text {-BOCS }}$ worst ever compulsion and greater $\mathrm{Y}$-BOCS worst ever total score, and diagnoses of OCD, any anxiety disorder, any BFRB, BDD, ADHD and substance abuse 
were all associated with increased risk of having an ED within a TTM population. This is consistent with Sallet et al.'s finding, which showed greater prevalence of anxiety disorders and BDD, in addition to SPD and ADHD (pre-Bonferroni correction), in an OCD and ED population vs. in an OCD population without comorbid EDs ${ }^{[15]}$. Greater Y-BOCS worst ever total scores, and greater prevalence of OCD and any anxiety disorder being associated with EDs in a TTM population was consistent with Zucker et al., who found that increased trait anxiety and compulsions (as part of the obsessive-compulsive spectrum) were associated with increased risk of TTM in an ED population [5]. As BFRBs and BDD are also obsessive-compulsive spectrum disorders, given the strong association between EDs and OCD, it is not surprising that they too were associated with an increased risk of having an ED in a TTM population. This also supports Sallet et al.'s finding that individuals with OCD and EDs had higher rates of BDD compared to those with only OCD 15$]$. It is interesting, though not surprising, that ADHD and substance abuse were both associated with increased risk of ED in TTM in the univariable analysis, as ADHD and substance abuse both represent the impulsive components on the impulsive-compulsive spectrum found in both EDs and TTM.

When the above factors were all compiled into a multivariable regression equation, it was determined that diagnosis of OCD and diagnosis of another BFRB (other than TTM) were associated with increased risk of ED in a TTM population. This finding is consistent with and a corollary to Keuthen et al.'s recent paper, which showed that a diagnosis of ED is associated with increased risk of OCD within a TTM population [20]. Is it also consistent with Fernández-Aranda et al., who showed that individuals with EDs (specifically binge eating) and impulse control disorders were more likely to have OCD symptoms than those with EDs without impulse control disorders $\stackrel{[21]}{\text {. }}$

It is interesting to note that it was the diagnoses that are part of the obsessive-compulsive spectrum disorders (OCD and an additional BFRB) that were ultimately significant in predicting increased risk of EDs in our TTM population. The marginal association between BDD and EDs in TTM may have been due to the low prevalence of BDD in general in our sample. These findings further support the relatedness between EDs and OCSDs, including BFRBs, and support the idea of EDs being on the obsessivecompulsive disorder spectrum. This finding is also consistent with Monzani et al.'s recent report of a shared heritability between all of the obsessive-compulsive and related disorders including, OCD, BDD, hoarding, skin picking and hair pulling ${ }^{[10]}$. It would be interesting for future research to examine the heritability factors of OCSDs and EDs together.

It is important to note study limitations. First, this sample included only females. Replication of the findings in future studies that include males is warranted, especially as gender itself may likely be a predictor of EDs in a TTM population. Also, there were differences in exclusion criteria and measures across sites, which could add heterogeneity to the analyses. Although this study utilized a very large sample size, future research would benefit from even larger samples in order to increase the power to detect associations with more modest effect sizes, and similarly to estimate the observed associations with more accuracy.

An additional limitation is that because this study was conducted in adults, one has to be careful about generalizing any findings to adolescents or other age groups. However, given that the most common age of onset of TTM is early adolescence, and the symptoms/course in adolescence continues into and 
is akin to that in adulthood [4], [11], one may be able to prudently apply some of these findings when working with adolescent patients. That said, conducting a similar study with adolescent patients would be very helpful in further examining the relatedness between eating disorders and TTM. Additionally regarding limitations, all ED diagnoses were grouped together in this study. This approach is common, especially given the limited number of subjects with EDs, though future studies would benefit from examining which factors may be predictive of specific EDs given the known differences between anorexia nervosa, bulimia nervosa and binge eating disorder. It is also important to note that Eating Disorder - Not Otherwise Specified (ED-NOS), DSM-IV diagnosis, was not screened for in the treatment studies analyzed. Given that ED-NOS was known to be a very common condition, it is possible that estimate of those with EDs in a TTM population is higher $[36]$. Future studies utilizing DSM-5 ED criteria (including Avoidant/Restrictive Food Intake Disorder) should help ameliorate that problem. Finally, it is important to note that our cohort consisted of participants in TTM research studies, and that they therefore may not accurately represent the TTM population as a whole. Thus, it is possible that our findings may not generalize to the entire TTM community. For future research, it would be helpful to conduct analyses in community/non-treatment seeking samples.

These findings highlight the importance of assessing for comorbid OCD and additional BFRBs in those with TTM when considering which patients may be most at risk for EDs. Compared to individuals with just $O C D$, those with $O C D$ and EDs are more likely to have higher lifetime prevalence of comorbid conditions, higher anxiety and depression scores, and higher frequency of suicide attempts [15]. Additionally, even after controlling for subtype of ED, the presence of an impulse-control disorder is associated with greater severity of ED and worse general psychiatric morbidity and psychopathology [21]. These findings ultimately will allow clinicians to identify which of their patients with TTM may be at greatest risk and have greatest psychiatric burden given that patients with EDs may not reveal their condition secondary to shame and/or are disinclined to seek treatment 225$]$.

Further research is needed to identify additional predictors of comorbid disorders and to better understand the complex relationships between BFRBs, OCD and EDs. This study should help to direct future research on the relationship between TTM and EDs, and help inform pathophysiology, phenomenology and treatment models.

\section{Conclusion}

In this trichotillomania cohort of 555 adult females, 7.2\% had a (lifetime) eating disorder diagnosis, including anorexia nervosa, bulimia nervosa and/or binge eating disorder. Using multivariable regression, we determined that having OCD and having an additional body-focused repetitive behavior disorder (such as skin-picking disorder or severe nail-biting), was associated with increased risk of having an eating disorder within a trichotillomania population. This study helps support the relationship between obsessive-compulsive spectrum disorders and eating disorders, and can help physicians identify patients at increased risk for eating disorders, a dangerous psychiatric condition. 


\section{Financial disclosures}

Dr. Keuthen receives royalties from New Harbinger, Inc. and has equity interest in Johnson \& Johnson, Merck \& Co., Inc., Pfizer, Inc., and Procter \& Gamble Co. She is vice-chair of the scientific advisory board of the TLC Foundation for Body-Focused Repetitive Behaviors. Dr. Grant has received research grants from NIMH, NIDA, National Center for Responsible Gaming, Brainsway, Forest, Takeda, and Psyadon Pharmaceuticals. He receives yearly compensation from Springer Publishing for acting as Editor-in-Chief of the Journal of Gambling Studies and has received royalties from Oxford University Press, American Psychiatric Publishing, Inc., Norton Press, and McGraw Hill. Dr. Scharf is a member of the Scientific Advisory Board of the Tourette Association of America and the TLC Foundation for BFRBs, has received research grants from the Tourette Association of America, and has received consulting fees from Nuvelation Pharma. Drs. Grant, Keuthen, and Scharf have received research grants from the TLC Foundation for Body-Focused Repetitive Behaviors. Dr. Woods has received research grants from $\mathrm{NIMH}$, and receives royalties from Oxford University Press. Dr. Stein has received research grants and/or consultancy honoraria from AMBRF, Biocodex, Cipla, Lundbeck, National Responsible Gambling Foundation, Novartis, Servier, and Sun. Ms. Curley, Mrs. Tung, Ms. Redden, Dr. Greenberg, and Dr. Lochner report no financial relationships with commercial interests.

\section{Role of funding sources}

We gratefully recognize financial support for this project from the TLC Foundation for Body-Focused Repetitive Behaviors and its BFRB Precision Medicine Initiative, as well as the Greater Kansas City Foundation.

\section{Contributions}

NK and EG designed the study. EC, JS and EG undertook the statistical analyses. NK, JG, CL, DW, DS, ET, SR, EC were involved in data management. EG wrote the first draft of the manuscript. All authors contributed to and have approved the final manuscript.

\section{Acknowledgment}

We also wish to express gratitude to Amanda Falcon, Miriam Frank, Zachary Madison, Naomi Stapleton, and Jacqueline Kenitz, who performed data input and cleaning, and Jennifer Fehring who helped with citations.

\section{References}

[1] American Psychiatric Association Diagnostic and statistical manual of mental disorders (5th ed.), American Psychiatric Association, Arlington, VA (2013)

[2] American Psychiatric Association Diagnostic and statistical manual of mental disorders (4th ed.), Author, Washington, DC (2000) 
[3] G.A. Christenson, R.L. Pyle, J.E. Mitchell Estimated lifetime prevalence of trichotillomania in college students J Clin Psychiatry, 52 (1991), pp. 415-417

[4] D.C. Duke, M.L. Keeley, G.R. Geffken, E.A. Storch Trichotillomania: a current review Clin Psychol Rev, 30 (2010), pp. 181-193

[5] N. Zucker, A. Von Holle, L.M. Thornton, M. Strober, K. Plotnicov, K.L. Klump, et al. The significance of repetitive hair-pulling behaviors in eating disorders J Clin Psychol, 67 (2011), pp. 391-403

[6] M.A. Richter, L.J. Summerfeldt, M.M. Antony, R.P. Swinson Obsessive-compulsive spectrum conditions in obsessive-compulsive disorder and other anxiety disorders Depress Anxiety, 18 (2003), pp. 118-127

[7] E. Hollander, S. Kim, A. Braun, D. Simeon, J. Zohar Cross-cutting issues and future directions for the OCD spectrum Psychiatry Res, 170 (2009), pp. 3-6

[8] K.A. Phillips, D.J. Stein, S.L. Rauch, E. Hollander, B.A. Fallon, A. Barsky, et al. Should an obsessivecompulsive spectrum grouping of disorders be included in DSM-V? Depress Anxiety, 27 (2010), pp. 528-555

[9] D.C. Houghton, J. Maas, M.P. Twohig, S.M. Saunders, S.N. Compton, A.M. Neal-Barnett, et al. Comorbidity and quality of life in adults with hair pulling disorder Psychiatry Res, 239 (2016), pp. $12-19$

[10] B. Monzani, F. Rijsdijk, J. Harris, D. Mataix-Cols The structure of genetic and environmental risk factors for dimensional representations of DSM-5 obsessive-compulsive spectrum disorders JAMA Psychiat, 71 (2014), pp. 182-189

[11] G.A. Christenson, T.B. Mackenzie, J.E. Mitchell Characteristics of 60 adult chronic hair pullers Am J Psychiatry, 148 (1991), pp. 365-370

[12] I. Snorrason, E.L. Belleau, D.W. Woods How related are hair pulling disorder (trichotillomania) and skin picking disorder? A review of evidence for comorbidity, similarities and shared etiology Clin Psychol Rev, 32 (2012), pp. 618-629

[13] C.M. Bulik, P.F. Sullivan, T.E. Weltzin, W.H. Kaye Temperament in eating disorders Int J Eat Disord, 17 (1995), pp. 251-261

[14] K.M. O'Brien, N.K. Vincent Psychiatric comorbidity in anorexia and bulimia nervosa: nature, prevalence, and causal relationships Clin Psychol Rev, 23 (2003), pp. 57-74

[15] P.C. Sallet, P.G. de Alvarenga, Y. Ferrao, M.A. de Mathis, A.R. Torres, A. Marques, et al. Eating disorders in patients with obsessive-compulsive disorder: prevalence and clinical correlates Int J Eat Disord, 43 (2010), pp. 315-325

[16] L. Bellodi, M.C. Cavallini, S. Bertelli, D. Chiapparino, C. Riboldi, E. Smeraldi Morbidity risk for obsessive-compulsive spectrum disorders in first-degree relatives of patients with eating disorders Am J Psychiatry, 158 (2001), pp. 563-569

[17] M. Cederlof, L.M. Thornton, J. Baker, P. Lichtenstein, H. Larsson, C. Ruck, et al. Etiological overlap between obsessive-compulsive disorder and anorexia nervosa: a longitudinal cohort, multigenerational family and twin study World Psychiatry, 14 (2015), pp. 333-338

[18] C.L. Boisseau, H. Thompson-Brenner, C. Caldwell-Harris, E. Pratt, T. Farchione, D.H. Barlow Behavioral and cognitive impulsivity in obsessive-compulsive disorder and eating disorders Psychiatry Res, 200 (2012), pp. 1062-1066 
[19] S. Jimenez-Murcia, F. Fernandez-Aranda, R.M. Raich, P. Alonso, I. Krug, N. Jaurrieta, et al.

Obsessive-compulsive and eating disorders: comparison of clinical and personality features Psychiatry Clin Neurosci, 61 (2007), pp. 385-391

[20] N.J. Keuthen, E.E. Curley, J.M. Scharf, D.W. Woods, C. Lochner, D.J. Stein, et al. Predictors of comorbid obsessive-compulsive disorder and skin-picking disorder in trichotillomania Ann Clin Psychiatry, 28 (2016), pp. 280-288

[21] F. Fernandez-Aranda, S. Jimenez-Murcia, E.M. Alvarez-Moya, R. Granero, J. Vallejo, C.M. Bulik Impulse control disorders in eating disorders: clinical and therapeutic implications Compr Psychiatry, 47 (2006), pp. 482-488

[22] C. Kisa, S.G. Yildirim, E. Goka Impulsivity and mental disorders Turk Psikiyatri Derg, 16 (2005), pp. 46-54

[23] L.A. Winkler, E. Christiansen, M.B. Lichtenstein, N.B. Hansen, N. Bilenberg, R.K. Stoving Quality of life in eating disorders: a meta-analysis Psychiatry Res, 219 (2014), pp. 1-9

[24] F.R. Smink, D. van Hoeken, H.W. Hoek Epidemiology of eating disorders: incidence, prevalence and mortality rates Curr Psychiatry Rep, 14 (2012), pp. 406-414

[25] A.E. Becker, K.T. Eddy, A. Perloe Clarifying criteria for cognitive signs and symptoms for eating disorders in DSM-V Int J Eat Disord, 42 (2009), pp. 611-619

[26] U.S. Department of Health \& Human Services Guidance regarding methods for de-identification of protected health information in accordance with the Health Insurance Portability and Accountability Act (HIPAA) privacy rule (2012)

[27] M.B. First, R.L. Spitzer, M. Gibbon, J.B. Williams Structured clinical interview for DSM-IV-TR axis I disorders-non-patient edition New York State Psychiatric Institute, New York (2001)

[28] J. Kaufman, B. Birmaher, D. Brent, U. Rao, C. Flynn, P. Moreci, et al. Schedule for affective disorders and schizophrenia for school-age children-present and lifetime version (K-SADS-PL): initial reliability and validity data J Am Acad Child Adolesc Psychiatry, 36 (1997), pp. 980-988

[29] B.O. Rothbaum, P.T. Ninan The assessment of trichotillomania Behav Res Ther, 32 (1994), pp. 651662

[30] Keuthen NJ, Stewart SE. Skin picking diagnostic inventory. [Unpublished Inventory].

[31] S.M. Darrow, C. Illmann, C. Gauvin, L. Osiecki, C.A. Egan, E. Greenberg, et al. Web-based phenotyping for Tourette syndrome: reliability of common co-morbid diagnoses Psychiatry Res, 228 (2015), pp. 816-825

[32] D.L. Pauls, C.R. Hurst Schedule for Tourette's syndrome and other behavioral syndromes Yale University Child Study Center, New Haven, CT (1987)

[33] D.L. Pauls, J.P. Alsobrook II, W. Goodman, S. Rasmussen, J.F. Leckman A family study of obsessivecompulsive disorder Am J Psychiatry, 152 (1995), pp. 76-84

[34] A.T. Beck, N. Epstein, G. Brown, R.A. Steer An inventory for measuring clinical anxiety: psychometric properties J Consult Clin Psychol, 56 (1988), pp. 893-897

[35] IBM Corporation IBM SPSS statistics for windows (20.0 ed.), Armonk, NY, IBM Corp. (2011)

[36] F.R. Smink, D. van Hoeken, H.W. Hoek Epidemiology, course, and outcome of eating disorders Curr Opin Psychiatry, 26 (2013), pp. 543-548 
